\title{
Natural Language Dialogue Service for Appointment Scheduling Agents*
}

\author{
Stephan Busemann, Thierry Declerck, Abdel Kader Diagne, \\ Luca Dini, Judith Klein, Sven Schmeier \\ DFKI GmbH \\ Stuhlsatzenhausweg 3, 66123 Saarbrücken, Germany \\ busemann@dfki.uni-sb.de
}

\begin{abstract}
Appointment scheduling is a problem faced daily by many individuals and organizations. Cooperating agent systems have been developed to partially automate this task. In order to extend the circle of participants as far as possible we advocate the use of natural language transmitted by email. We describe Cosma, a fully implemented German language server for existing appointment scheduling agent systems. Cosma can cope with multiple dialogues in parallel, and accounts for differences in dialogue behaviour between human and machine agents. NL coverage of the sublanguage is achieved through both corpusbased grammar development and the use of message extraction techniques.
\end{abstract}

\section{Motivation}

Appointment scheduling is a problem faced daily by many individuals and organizations, and typically solved using communication in natural language (NL) by phone, fax or by mail. In general, cooperative interaction between several participants is required. Since appointments are often scheduled only after a sequence of point-to-point connections this will, at times, necessitate repeated rounds of communication until all participants agree to some date and place. This is a very time-consuming task that should be automated.

Systems available on the market allow for calendar and contact management. As (Busemann and Merget, 1995) point out in a market survey, all planning and scheduling activity remains with the user. Cooperative agent systems developed in the field of Distributed AI are designed to account for the scheduling tasks. Using distributed rather than centralized

\footnotetext{
* This work has been supported by a grant from the German Federal Ministry of Education, Science, Research and Technology (FKZ ITW-9402).
}

calendar systems, they not only guarantee a maximum privacy of calendar information but also offer their services to members or employees in external organizations. Although agent systems allow users to automate their scheduling tasks to a considerable degree, the circle of participants remains restricted to users with compatible systems.

To overcome this drawback we have designed and implemented CosmA, a novel kind of NL dialogue systems that serves as a German language frontend system to scheduling agents. Human language makes agent services available to a much broader public. Cosma allows human and machine agents to participate in appointment scheduling dialogues via e-mail. We are concerned with meetings all participants should attend and the date of which is negotiable.

\section{Design guidelines}

Cosma is organized as a client/server architecture. The server offers NL dialogue service to multiple client agent systems. Up to now, three different types of agent systems have been hooked up to the NL server. Agents developed in-house were used for the early system described in (Busemann et al., 1994). In a subsequent version, the MEKKA agents developed by Siemens AG (Lux et al., 1992) have been adapted. We present in Section 4 a third kind of client system, the PASHA II user agent.

Given the use of distributed calendar systems, techniques used by both human and machine agents for cooperatively scheduling appointments must be based on negotiation dialogues. However, human dialogue behaviour differs from interaction between machine agents considerably, as will be discussed in Section 4. A human-machine interface to existing appointment scheduling agent systems should comply to the following requirements:

- Human utterances must be analyzed to correspond closely to agent actions.

- Machine utterances must conform to human dialogue strategies. 
Artificial communication languages have been designed for human discourse, e.g. (Sidner, 1994), as well as for agent-agent interaction, e.g. (Steiner et al., 1995). What would be needed for Cosma is a mapping between strategies implemented in such languages. Since the type of agent system connected to the Cosma server is not restricted by its dialogue behaviour, preference was given to implement application-dependent mappings instead of developing a generic formalism. As a consequence, CosMA operates with general and reusable processing modules that interpret domain- and task-specific data.

The same principle was also adopted for NL analysis. The server must analyze human-generated text and verbalize machine-initiated goals. For a plausible application, the server must be:

- complete with respect to a sublanguage: all relevant information related to appointments must be analyzed,

- sufficiently robust to deal with inconsistent analysis results.

Within the HPSG-based approach to grammar description adopted for the early system (Uszkoreit et al., 1994), achieving these goals turned out to be difficult. This "deep" approach to NLU describes NL expressions at general linguistic levels (syntax and surface semantics), and attempts to capture the complete meanings of all and only the grammatical sentences. However, an NL system in a realistic application should not fail on unexpected input. Moreover, the surface semantic representations derived by the grammar were too close to NL for an agent system to deal with.

With the present version of the NL server these problems are solved by adopting a "shallow" analysis approach, which extracts meanings from those portions of a text that are defined as interesting and represents them in an agent-oriented way. Instead of failing on unexpected input, shallow parsing methods always yield results, although they may not capture all of the meaning intended by the user. By just describing the verbalizations of relevant information, shallow parsing grammars are highly domain-specific and task-oriented. In COSMA, shallow analysis is divided up into an application of the message extraction component smes (discussed in Section 5) and a semantic analysis component IMAS (Section 6). The former extracts appointment-related information from users' input texts. It is based on finitestate automata that were defined with help of an annotated corpus of e-mail messages. The task of the latter is to derive a client-oriented semantic representation, including the communicative intention and the complete specification of time points needed, which is based on context and semantic inferences.

The robustness requirement is fulfilled by recognizing failures within the server during semantic analysis, and possibly within the client systems, and by clarification dialogues (cf. Section 6.1).

After an overview of generation in Cosma (Section 7) we discuss component interaction in Section 8. A novel type of object-oriented architecture is needed to treat multiple dialogues in parallel. Virtual partial system instances are maintained as long as a dialogue is going on. One such instance is shown in Figure 1.

\section{A complete sample dialogue}

A complete sample dialogue taken from the system's present performance will serve as a reference throughout the paper. Every utterance is numbered and labeled; the labels indicate speakers. We assume a three-party e-mail negotiation between a human $(H)$, who does not use a scheduling agent system, and two machine agents (A, B) that schedule appointments for their respective owners. In the server, human interactions with multiple machine partners are treated as different NL dialogues (in the present case between $\mathrm{H}$ and $\mathrm{A}$, and $\mathrm{H}$ and $\mathrm{B}$ ). In what follows, $\mathrm{H}$ is the initiator, but Cosma also copes with machine-initiated dialogues (cf. Section 4). ${ }^{1}$

(01) Ich würde Sie gern am Montag, dem 2.11.96 $\mathrm{H}$ wegen der bevorstehenden Projektbegutachtung treffen. [I would like to meet you on Monday Nov. 21996 about the upcoming project review.]

(02) COSMA hat die folgende Zeitangabe verA, B standen, die nicht konsistent ist: Montag, den 2. 11. 1996. Könnten Sie bitte den Wochentag oder das Datum korrigieren? [COSMA has understood the following time expression, which is not consistent: Monday, Nov. 2 1996. Could you please correct the weekday or the date?]

(03) Ich meinte natürlich Montag den 4. 11. [I $\mathrm{H}$ meant of course Monday, Nov. 4.]

(04) Am 4. 11. 1996 paßt es bei mir zwischen 13 A und 18 Uhr. [Nov. 4, 1996 would suit me between 1 and 6 p.m.]

(05) Leider kann ich am 4.11. 1996 nicht kommen. B [Unfortunately I can't come on Nov. 4 1996.]

(06) Der vorige Vorschlag wurde abgelehnt. $\mathrm{H}$ Können wir uns stattdessen am Dienstag treffen? [The previous proposal was rejected. Can we meet instead on Tuesday?]

\footnotetext{
${ }^{1}$ Agent-agent interaction is based on a formal representation language rather than on NL.
} 


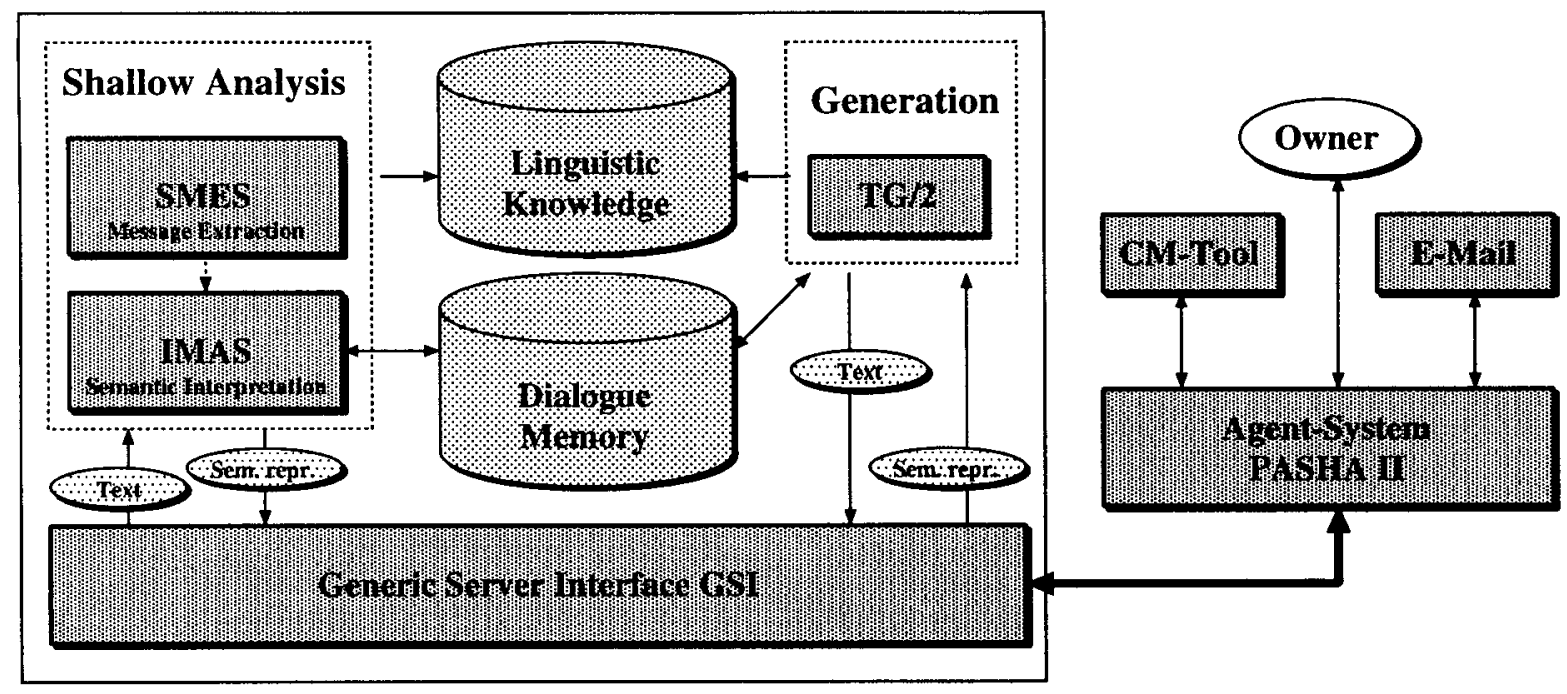

Figure 1: The Cosma architecture: a client connected to a server instance may issue requests to receive a semantic representation for a text, or to generate a text from a semantic representation. The generic server interface invokes the necessary server processes and maintains interaction with the client.

(07) Zu folgenden Zeiten geht es bei mir: am 5. A $\quad 11.1996$ zwischen 8 und $12 \mathrm{Uhr}$ und zwischen 16 und 18 Uhr. [The following times would suit me: Nov. 5,1996 between 8 and 12 a.m. and between 4 and 6 p.m.]

(08) Am 5. 11. 1996 paßt es bei mir zwischen 10 B und 12 Uhr. [Nov. 4, 1996 would suit me between 10 and 12 a.m.]

(09) Wie wäre es dann um 10? [How about 10 $\mathrm{H}$ then?]

(10) Ich sage den 5. 11. 1996 um 10 Uhr zu. [I A, B accept Nov. 5, 1996 at 10 a.m.]

(11) Das Treffen wird also am 5. 11. 1996 um 10 $\mathrm{H}$ Uhr stattfinden. [The meeting will take place on Nov. 5, 1996 at 10 a.m.]

In (01) $\mathrm{H}$ sends by mistake an inconsistent temporal expression to $A$ and $B$, giving rise to clarification dialogues initiated by each of $A$ and $B(02)$. The repair provided by $\mathrm{H}(03)$ is underspecified with respect to clock time (see also (06)), hence the agents offer free time slots in accordance to their calendars $(04,07,08)$. These time slots are blocked until further notice. ${ }^{2}$ Since B rejects the proposed date (05), a new loop is started by $H(06)$. When $H$ notices that Tuesday is promising, she chooses to refine her proposal by suggesting a clock time (09). Dynamic context knowledge allows the server to reconstruct a full time specification that is interpreted by the agents as an alternative proposal. Refinements can

\footnotetext{
${ }^{2}$ Cancellations of rescrved slots due to a high-priority request are a straight-forward extension of the present coverage.
}

thus be dealt with completely in the server, whereas the agents may or may not have a concept of refinement. After all agents accept a proposal, the date is confirmed by the initiator (11). Upon receipt of the confirmation, the agents fix the date in their calendars. Server and agents consider the dialogues as completed.

\section{Dialoging scheduling agents}

\subsection{The PASHA II system}

PASHA II agents (Schmeier and Schupeta, 1996) are designed according to the InterRaP agent architecture (Fischer et al., 1995), a layer-based agent model that combines deliberative and reactive behaviour. The "heart" of an agent is the cooperative planning layer, in which negotiation strategies are represented as programs and executed by a language interpreter. This supports easy modification and exchange of plans. The local planning layer consists of a constraint planner which reasons about time slots in the agent's (i.e. its owner's) calendar. In contrast to the planning layers, the behaviour-based layer consists of the agent's basic reactive behaviour and its procedural knowledge. The world interface realizes the agent's sensing and acting capabilities as well as the connection to its owner. PASHA II agents are connected to the Unix CM calendar management tool, but can easily be hooked up to other calendar systems.

PASHA II agents are easily adapted to the owner's preferences. For instance, any time slots the owner does not wish the agent to use can be blocked. By virtue of this mechanism, a working day could be defined as an interval from e.g. 8 a.m. until 6 p.m. 
except for Saturdays, Sundays and holidays. Moreover, gaps between appointments may be specified in order to permit sufficient time between meetings.

\subsection{Adapting agents to the Cosma server}

Taking PASHA II as a representative, we describe the requirements for an agent system to connect to the Cosma server.

Interface to the server. The four main modules include the basic TCP/IP connection to the server; a parser of semantic representations of the server's analysis results, which yields PASHA II structures; an instantiation mechanism for semantic generation templates; and a control regime that keeps track of the current dialogue. The control regime confirms results of the server, or it activates the server's backtrack mechanism if the semantic representation received does not fit within the current dialogue step, or it issues a request for repair if backtracking should not yield any further results.

Receiving and sending e-mail. The PASHA II interaction mechanism includes, besides communication via TCP/IP protocols, e-mail interaction. The agent may poll its owner's mailbox or have one of its own. Either the agent or its owner is referred to as actor in the agent's e-mail messages (see Section 7).

Dialogue behaviour. An agent has to generate and understand different dialogue actions represented by corresponding cooperation primitives such as proposing, accepting, rejecting, canceling or fixing a meeting (Steiner et al., 1995).

Agent-agent interaction usually relies on an initiating agent being responsible for the success of a negotiation. The initiator's broadcast proposal is triggered by its owner, who determines partners, duration and an interval within which the appointment should be scheduled. The agent proposes the first slot in the interval that is available according to its calendar. In case of a rejection of one or more participants, the initiator would continue to propose new time slots to all partners until everyone agrees to a common date or there is no such slot within the interval. Note that in case of rejection (see (05)) PASHA II agents do not use counter-suggestions.

In human-human negotiation, efficiency is a major goal. Humans often follow the least effort principle (Dahlbäck, 1992): the initiator broadcasts a proposal including a time interval within which the meeting should take place (e.g. (03)) and expects refinements or counter-proposals from the participants. As the example shows this may imply the use of underspecified temporal descriptions. This strategy requires less communication because a greater amount of information is exchanged in one dialogue step between the participants.

Handling underspecified temporal information by offering free time slots (see (04), (07), and (08)) is among the extensions of PASHA II at the local planning layer. Note that this strategy can be instanti- ated in different ways, as becomes clear from dealing with expression such as next week: Only a selection of free time slots can be provided here, which is explicitly marked using e.g. for instance. Moreover, we consider it indispensable to have agents understand and generate counter-proposals to avoid inefficient plain rejections like (05).

\section{Covering the domain language}

\subsection{Corpus-based annotation}

In order to determine the coverage of the sublanguage relevant for the application and to measure progress during system development, a corpus of 160 e-mails was selected as reference material from several hundred e-mails collected from the domain of appointment scheduling. The e-mails were manually analyzed and annotated with major syntactic and semantic features as well as speechact information. A combination of two relational database systems was employed to ease the storage, maintenance, extension and retrieval of the NL data:

(i) DiTo (Nerbonne et al., 1993), a full text database where the e-mails can be accessed,

(ii) tsdb (Oepen et al., 1995), an elaborated fact database which permits the extraction of specific linguistic constructions together with the associated linguistic annotations. ${ }^{3}$

\begin{tabular}{|l|l|}
\hline Annotation & Example \\
\hline \hline Prepositional Phrases: Wie wäre es [How about] ... \\
\hline PP_temp & in dieser Woche? [in this week?] \\
PP_temp-date & am 4.11? [on the 4th of Nov.?] \\
PP_temp-day & am Montag? [on Monday?] \\
PP_temp-dur & von 8 bis 12? [from 8 to 12?] \\
PP_temp-time & um 10? [at 10?] \\
\hline Noun Phrases: Ich komme [I come] ... \\
\hline NP_temp & zwei Stunden später. \\
& [two hours later.] \\
NP_temp-date & am Montag, den 4.11. \\
& [on Monday, the 4th of Nov.] \\
NP_temp-day & Montag, 14 h. [Monday, 2 pm.] \\
NP_temp-time & Montag, 14 h. [Monday, 2 pm.] \\
\hline
\end{tabular}

Figure 2: Semantic annotation of PPs and NPs (annotated linguistic material in italics)

The annotation work is based on the TSNLP framework (Lehmann et al., 1996) where detailed category and function lists are defined for the structural and dependency structure annotation of linguistic material for NLP test suites. For Cosma, the classification has been extended according to semantic information relevant for the appointment domain. For instance, PPs and NPs were specified further, introducing a more fine-grained semantic anno-

\footnotetext{
${ }^{3}$ DiTo and tsdb entries are linked via c-mail identifiers.
} 
tation for temporal expressions, as is shown in Figure 2.

The results of database queries provided valuable insights into the range of linguistic phenomena the parsing system must cope with in the domain at hand. Grammar development is guided by a frequency-based priority scheme: The most important area - temporal expressions of various categories - followed by basic phenomena including different verbal subcategorizations, local and thematic PPs, and the verbal complex are successfully covered.

\subsection{Message extraction with smes}

The message extraction system smes (Neumann et al., 1997) is a core engine for shallow processing with a highly modular architecture. Given an ASCII text, smes currently produces predicate argument structures containing shallow semantic analyses of PPs and NPs. The core of the system consists of:

- a tokenizer, which scans the input using a set of regular expressions to identify the fragment patterns (e.g. words, date expressions, etc.),

- a fast lexical and morphological processing of 1,5 million German word forms,

- a shallow parsing module based on a set of finite state transducers,

- a result combination and output presentation component.

Based on the information delivered by the morphological analysis of the identified fragment patterns, the system performs a constituent analysis. In order to combine complements and adjuncts into predicate-argument structures, special automata for verbs are then activated over the sequence of constituents analyzed so far. Starting from the main verb $^{4}$, a bidirectional search is performed whose domain is restricted by special clause markers. smes output yields information about the utterance relevant for the subsequent semantic analysis.

\subsection{Semi-automatic grammar development}

The concrete realization of the automata is based on the linguistic annotations of the e-mail fragments in the corpus. The annotations render a semiautomatic description of automata possible. For instance, verb classification directly leads to the lexical assignment of a corresponding automaton in smes. By deriving parts of the grammar directly from corpus annotations, maintenance and extension of the grammars are eased considerably.

On the other hand, corpus extension can be supported by smes analyses. Existing automata can be

\footnotetext{
"If no verb is found, a "dummy" entry triggers processing of verbless expressions, which occur frequently in c-mail communication.
}

used to annotate new material with available linguistic information. Manual checking of the results reveals gaps in the coverage and leads to further refinement and extension of the automata by the grammar writer.

This way, grammar development can be achieved in subsequent feedback cycles between the annotated corpus and smes automata. The implementation of the annotation procedure based on the smes output format is underway.

\section{Semantic interpretation}

Semantic representations produced by smes are mapped into a format suitable for the PASHA-II client by the IMAS component (Information extraction Module for Appointment Scheduling). IMAS is based on a domain-dependent view of semantic interpretation: information-gathering rules explore the input structure in order to collect all and only the relevant information; the resulting pieces of information are combined and enriched in a monotonic, non-compositional way, thereby obtaining an IL (Interface Level) expression, which can be interpreted by the agent systems. In spite of the noncompositionality of this process, the resulting expressions have a clear model-theoretic interpretation and could be used by any system accepting first order logic representations as input.

IL expressions have been designed with the goal of representing both a domain action that is easily mapped onto an agent system's cooperation primitive, and the associated temporal information, which should be fully specified due to contextual knowledge. Temporal information is partitioned into RANGE, APPOINTMENT and DURATION information. RANGE denotes the interval within which a certain appointment has to take place (e.g. in (03)). APPOINTMENT denotes the interval of the appointment proper (e.g. in (10)). Intervals in general are represented by their boundaries. DURATION, on the contrary, encodes the duration of the appointment expressed in minutes. The backbone of an IL expression is thus the following:

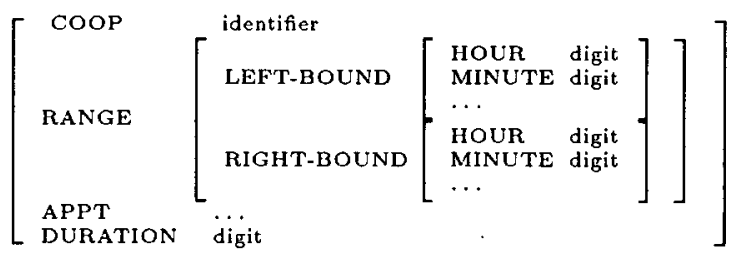

IMAS relies on three basic data structures. The sentence structure contains all the IL expressions obtained from the analysis of a single sentence. They are ranked according to their informativeness.

The text structure contains all the sentence structures obtained from the analysis of a whole message. Here ranking depends not only on informative- 
ness but also on "dialogue expectation": sentence structures are favoured that contain a domain action compatible with the IL expression previously stored in the discourse memory. As a result, the NL server will pass to the client the most informative IL expression of the most informative and contextually most relevant sentence of the analyzed text. ${ }^{5}$

The discourse memory is structured as a sequence containing all information collected during the dialogue. Thus it contains both IL expressions committed by the client and semantic input structures from generation. The discourse memory is used by IMAS as a stack.-

The procedural core of IMAS is represented by the transformation of the input smes representation into a set of IL expressions. This process is organized into three steps:

Linguistic extraction. The semantic representation of the input smes structure is explored by a set of rules in such a way that all information relevant for the appointment domain is captured. For every type of information (e.g. domain action, hour of appointment, duration, etc.) a different set of rules is used. The rules are coded in a transparent and declarative language that allows for a (possibly underspecified) description of the smes input (represented as a feature structure) with its associated "information gathering" action.

Anchoring. Most utterances concerning the domain of appointment scheduling are incomplete at least in two respects. Either they contain expressions which need to be delimited in order to be pragmatically plausible (underspecification, e.g. (09)), or they refer to intervals which are not explicitly mentioned in the sentence (temporal anaphora). The first class includes probably any NL time expression; even a simple expression such as (01) requires some extralinguistic knowledge to be understood in its proper contextual meaning (in (01) the "working day" interval of the respective day must be known). The reconstruction of underspecified temporal expressions is performed by a set of template filling functions which make use of parameters specified by the client system at the beginning of the dialogue.

Temporal anaphora include expressions such as on Monday, tomorrow, next month, whose interpretation depends on the discourse context. Solving anaphoric and deictic relations involves a rather complex machinery which borrows many concepts from Discourse Representation Theory. In particular, we assume a procedure according to which the antecedent of an anaphoric temporal expression is first looked up in the IL expressions of the text already parsed (with a preference for the most recent expressions); if no one is found, the discourse memory is consulted to retrieve from previous parts of the

\footnotetext{
${ }^{5}$ If the client is not satisfied with such an expression, backtracking will pass the next-best structure etc.
}

dialogue a temporal expression satisfying the constraints under analysis. If the search fails again, the expression is interpreted deictically, and resolved w.r.t. to the time the message was sent.

Inferences. IL expressions can be enriched and disambiguated by performing certain inferences involving temporal reasoning. Besides trivial cases of temporal constraint resolution, such as guessing the endpoint of an appointment from its startpoint and its duration, our inference engine performs disambiguation of domain actions by comparing intervals referred to by different dialogue utterances. For instance, if an utterance $u$ describing an interval $I$ is ambiguous between a refinement and a modification and the previous utterance refers to an interval $J$ including $I$, then $u$ can be disambiguated safely as denoting a refinement. Analogous inferences are drawn by just checking the possible combinations of domain actions across the current dialogue (a rejection can hardly be followed by another cancellation, a fixing cannot occur after a rejection, etc.). The constraints guiding this disambiguation procedure are encoded as filters on the output of IMAS and reduce the set of pragmatically adequate IL expressions.

\subsection{Handling of analysis failures}

Sometimes IMAS produces an output which cannot be used by the PASHA-II client. This happens when the human message is either too vague (What about a meeting?), or contains an inconsistent temporal specification (as in (01)). In these cases IMAS stores the available information, and the server generates a request for clarification in order to recover the necessary temporal specifications or to fix the already available ones. This request is mailed to the human partner. It includes the list of misspelled words found in the input message, which may give the partner a clue for understanding the source of the error. Once a clarification is provided, the server attempts to build an IL expression by merging and/or replacing the information already available with the newly extracted one (cf. (03)). If the resulting IL expression satisfies the constraints on well-formedness, it is shipped to the PASHA-II client. Otherwise the clarification subdialogue goes on along the same lines.

\section{Generation}

Client systems usually want to express in NL a cooperation primitive and a date expression. Hence NL generation is based on a semantic template filled by the client. Depending on its content the template is unified with a prefabricated structure specifying linguistic-oriented input to the generator. The same holds for failure messages, such as (02), and for specifications of free time slots, as in (07), where simple rules of aggregation take care not to repeat the full date specification for each clock time mentioned.

The production system TG/2 (Busemann, 1996) 
proved to be sufficiently flexible to accomplish this task by its ability to generate preferred formulations first. For instance, Cosma clients can parameterize TG/2 so as to refer to their owner by a first person pronoun or by a full name, or to use formal or informal form of addressing the human hearer, or to prefer deictic time descriptions over anaphorical ones.

\section{A novel architecture}

A NLP server which can both provide a range of natural language services and process multiple dialogues for a variety of applications in parallel requires (1) an architecture that ensures a high degre of reusability of NLP resources, (2) the availability of a robust interface that guarantees transparency and flexibility with respect to data representation and task specification, (3) client-driven server parametrization, (4) support for incremental, distributed and asynchronous robust data processing, and (5) advanced concepts for synchronization with respect to parallel dialogue processing for multiple clients. Due to the limited functionality of common architectural styles (Garlan and Shaw, 1993) with respect to these requirements, a novel object-oriented, manager-based and generic architecture has been designed and implemented. It combines techniques from different areas - in particular, from object technology (Booch, 1994) and from coordination theory including workflow management (Malone and Crowston, 1991) and is based on two main concepts: the cooperating managers approach (COCONUTS) and the virtual system architecture model.

\subsection{A manager-based approach}

Managers in the coconuTs model are control units which coordinate or perform specific activities and cooperate with each other in a client/server form. Their responsabilities, properties, behaviour and interface are determined by the classes they belong to. The prominent CoconUTS managers are: the data manager, which provides services related to representation, printing, conversion and transmission of data; the report manager, which supports specification, generation and printing of processing reports; the global interface manager, which provides a generic server interface; the computing components managers (CCMs), which encapsulates the system's components and let them appear as servers; and, finally, the workflow manager, which is the main control unit.

\subsection{Coordination and control}

Coordinating internal system activities with respect to parallel dialogue processing (including backtracking and failure recovery facilities) requires very powerful and flexible mechanisms for task scheduling, synchronization and control. In COCONUTS this task is carried out by the workflow manager, which also manages interdependencies between these activities while avoiding redundant ones and controlling the flow of work among the involved managers (e.g., passing subtasks from one manager to another in a correct sequence, ensuring that all fulfill their required contributions and taking default actions when necessary). The behaviour and function of the workflow manager are determined by the following sequence of operations: identifying and formulating a workflow goal, decomposing it into subgoals, determining and allocating resources for achieving the subgoals, elaborating and, eventually, executing an operation plan. It also provides a range of specialized exception handlers to ensure robustness (see Section 6.1).

\subsection{A generic server interface}

Flexible and reliable client/server communication is made possible by the generic server interface module GSI. It includes a declarative, feature-based representation and task specification language CCL and an object-oriented communication and data transfer module CCI. For CCL a parser, a printer and an inference engine are available. CCI contains various kinds of interface objects containing higher-level protocols and methods for reliable TCP/IP-based communication, data encoding/decoding and buffering, as well as priority and reference management. Note that interface objects are accessible through their TCP/IP-based internet addresses and can be associated to any component (cf. Figure 1). This way, subsystems can, on demand, be used as servers, e.g. smes or the generator.

\subsection{Integrating heterogenous components}

Each Cosma server component is encapsulated by a CCM (computing component manager), which makes its functionality available to other managers. A $\mathrm{CCM}$ has, among other things, a working (shortterm) memory, a long-term memory and a variety of buffers for storing and managing computed solutions for subsequent use. Using these features a CCM easily simulates incrementality and realizes intelligent backtracking by providing the computed solutions in a selective manner. A component can be released by a $\mathrm{CCM}$ it is bound to when the latter does no longer need its services; e.g. if the component has already computed all solutions. This permits efficient resource sharing, as several CcMs can be associated to one component. Thus, associating interface objects with CCMs provides a flexible way of realizing distributed processing performed by components implemented in different languages and running on different machines.

\subsection{The virtual system architecture}

The virtual system architecture allows for efficient parallel dialogue processing. It is based on the con- 
cept of cooperating object-oriented managers with the ability to define one-to-many relationships between components and ccMs. The key idea consists in adopting a manager-based/object-based view of the architecture shown in Figure 1. This architecture represents a virtual system (also called operation context), which is a highly complex object consisting of a variety of interacting managers. It may inherit from different classes of operation contexts, whose definitions are determined by the underlying domains of application. Thus, multiple dialogues are processed in parallel just by running each dialogue in a separate virtual system. As soon as a dialogue is completed, the assigned virtual system can be reused to process another one. Conceptually, no constraints are made on the number of active virtual systems in the server software. In order to ensure correct processing, a manager may operate in only one virtual system at a time. Note that managers can still be shared by virtual systems and they behaviour can vary from one system to another.

\section{Conclusion}

We described Cosma, a NL server system for existing machine agents in the domain of appointment scheduling. The server is implemented in Common Lisp and C. The PASHA II agent is implemented in DFKI-Oz (Smolka, 1995).

Robust analysis of human e-mail messages is achieved through message extraction techniques, corpus-based grammar development, and clientoriented semantic processing and representation. The virtual server architecture is a basis for the flexible use of heterogeneous NLP systems in real-world applications including, and going beyond, CosmA.

Future work includes extensive in-house tests that will provide valuable feedback about the performance of the system. Further development of CosMA into an industrial prototype is envisaged.

\section{References}

Grady Booch. 1994. Object-Oriented Analysis and Design with Applications. Benjamin/Cummings, Menlo Park.

Stephan Busemann and Iris Merget. 1995. Eine Untersuchung kommerzieller TerminverwaltungsSoftware im Hinblick auf die Kopplung mit natürlichsprachlichen Systemen. Technical Document D-95-11, DFKI, Saarbrücken.

Stephan Busemann et al. 1994. COSMAmulti-participant NL interaction for appointment scheduling. Technical Report RR-94-34, DFKI, Saarbrücken.

Stephan Busemann. 1996. Best-first surface realization. In Donia Scott, editor, Eighth International
Natural Language Generation Workshop. Proceedings, Herstmonceux, Univ. of Brighton.

Nils Dahlbäck. 1992. Representations of Discourse. Cognitive and Computational Aspects. Ph.D. thesis, Department of Computer and Information Science. Linköping University.

Klaus Fischer et al. 1995. Unifying control in a layered agent architecture. Technical Memo TM94-05, DFKI, Saarbrücken.

David Garlan and Mary Shaw. 1993. An introduction to software architecture. SEI-93-TR-033, Software Engineering Institute, Carnegie Mellon University, Pittsburg, Pennsylvania 15213.

Sabine Lehmann et al. 1996. TSLNP - Test Suites for Natural Language Processing. In Proceedings of COLING-96, pages 711-716, Copenhagen.

Andreas Lux et al. 1992. A Model for Supporting Human Computer Cooperation. In $A A A I$ Workshop on Cooperation among Heterogeneous Intelligent Systems, San Jose, Ca.

Thomas W. Malone and Kevin Crowston. 1991. Toward an interdisciplinary theory of coordination. Technical Report CCS TR 120, Center for Coordination Science, Sloan School of Management, MIT, Cambridge, MA.

John Nerbonne et al. 1993. A Diagnostic Tool for German Syntax. Machine Translation, 8(1-2).

Günter Neumann et al. 1997. An information extraction core system for real world German text processing. In this volume.

Stephan Oepen et al. 1995. The TSNLP database: From tsct(1) to tsdb(1). Report to LRE 62-089, DFKI.

Sven Schmeier and Achim Schupeta. 1996. Pasha II - a personal assistant fo scheduling appointments. In First Conference on Practical Application of Multi Agent Systems, London.

Candace L. Sidner. 1994. An artificial discourse language for collaborative negotiation. In Proc. 12th National Conference on Artificial Intelligence. Volume 1, pages 814-819, Seattle, WA.

Gert Smolka. 1995. The Oz Programming Model. Research Report RR-95-10, DFKI, Saarbrücken.

Donald Steiner et al. 1995. The conceptual framework of MAIL. In Cristiano Castelfranchi and Jean-Pierre Müller, editors, From Reaction to Cognition. 5th European Workshop on Modelling Autonomous Agents in a Multi-Agent World (MAAMAW 93), pages 217-230. Springer, LNAI, Vol. 957.

Hans Uszkoreit et al. 1994. DISCO-An HPSGbased NLP System and its Application for Appointment Scheduling. In Proceedings of COLING-94, Kyoto. 